\title{
Die Berufung evangelischer Theolog(i)en in Baden auf die Confessio Augustana vom 16. bis zum 19. Jahrhundert
}

\section{Einführung}

Wer sich mit wachen Augen im Brettener Melanchthonhaus bewegt, wird sehr schnell eines der Hauptbilder der Halle als Darstellung des Augsburger Reichstags von 1530 identifizieren. Kaiser und Stände sind zu erkennen, dazu die Übergabe eines Buches durch einen Mann in der Schaube des Gelehrten. Im Vordergrund ist eine hohe edle Gestalt zu erkennen, ebenfalls in der Gelehrtenschaube. Und auch wenn wir wissen, dass Philipp Melanchthon (1497-1560) keineswegs eine hohe Gestalt hatte, ist dem Künstler doch gelungen, ganz klar zu zeigen, wer hier steht: eben Philipp Melanchthon. Und auch wenn wir weiter wissen, dass er damals gar nicht dabei war, als ein kursächsisches „Buch“, (wenn man so will) „sein“ Buch er war ja Hauptverfasser - dem Kaiser übergeben wurde, hat sich der Künstler diese Darstellung erlaubt, nicht als Fehler, nicht als Missverständnis, sondern als künstlerische Interpretation. Der Genius Melanchthons, ja er selbst war da, quasi real präsent, als das für die weitere Geschichte des Protestantismus nachhaltigste Dokument in die Geschichte des Reiches eintrat: das sog. Augsburger Bekenntnis, lat. Confessio Augustana, abgekürzt: CA.

Die folgenden Ausführungen befassen sich nicht mit der Entstehungsgeschichte der CA, auch nicht mit den theologischen Fragen darin (auch wenn die Abendmahlslehre immer einmal anklingen muss). Vielmehr möchte ich zeigen, wie und warum eine Landeskirche, in diesem Fall die Evangelische Kirche in Baden, durch die Jahrhunderte hindurch durch die CA geprägt worden ist; oder vielleicht zurückhaltender und zugleich präziser formuliert: welche Rolle sie in der badischen evangelischen Kirche gespielt hat. Es wird sich zeigen, dass dies recht unterschiedliche Rollen gewesen sind und sich die CA als durchaus anpassungsfähig erwies, was ihre theologische Interpretationsfähigkeit oder auch politische Funktion betraf.

Eigentlich ist das gar nichts Besonderes, sondern vielleicht sogar ganz typisch für die CA, lange bevor sie die badische Geschichte bestimmte: Schon vor 40 Jahren hat Gottfried Seebaß gezeigt, dass und wie die CA aus einer Schrift zur Verteidigung evangelischer Reformen in den Gemeinden zu einem Bekenntnis 
wurde, das dann auch ausschließende Artikel kannte. ${ }^{1}$ Ja, das Changieren der eher apologetischen und der eher polemischen Aussagen in der CA ist sogar schon Teil ihrer Entstehungsgeschichte - gestaltet je nach dem religionspolitischen Klima vor dem Reichstag und dann in den Wochen des Reichstages selbst.

Die Frage, was die CA nun eigentlich sei: Diskussionsgrundlage, Arbeitspapier, Glaubenszeugnis oder Verteidigungsschrift, hat die Geschichte dann beantwortet. Die CA wurde das vor Kaiser und Reich von den evangelischen Ständen vorgetragene Bekenntnis der evangelischen Kirche. Neben seinem theologischen Gehalt und seinem Bekenntnischarakter wurde es 1555 aber auch zur Rechtsgrundlage für die lutherische Kirche. Nur die Signatare der CA standen - abgesehen von Altgläubigen - unter dem Schutz des Reichsfriedens, also die sog. Augsburger Religionsverwandten.

\section{Die Geltung der Confessio Augustana in der Markgrafschaft Baden-Pforzheim des Jahres 1556}

Es war der eben genannte Augsburger Friede von 1555, der den Markgrafen Karl II. (1529; reg. 1552-1577) bewog, für sein kleines Territorium zwischen Pforzheim, Mühlburg ${ }^{2}$ und Graben wie auch für das sog. Markgräflerland dem Drängen vor allem Württembergs zu folgen und die Reformation einzuführen. Angesichts der damals schwelenden Streitigkeiten im Protestantismus waren zwei Optionen möglich.

Zum einen wurde Baden-Pforzheim (seit 1565 Baden-Durlach) von zwei Seiten umworben. Da war das nahe Württemberg mit seiner bedeutenden Universität in Tübingen, das damals noch ein eher mildes Luthertum vertrat, was sich aber zwischen 1559 und 1563 änderte. Da waren aber auch herzoglich-sächsische Theologen Weimarer und Jenenser Prägung, die den Anspruch vertraten, die Lehre Luthers v.a. im Abendmahl reiner zu vertreten, als Melanchthon spätestens seit 1540 und intensiviert in den 1550er-Jahren es tat.

Zum andern: Lehrgrundlage war die CA, sie musste es sein, um die Reformation überhaupt reichsrechtlich unbeanstandet durchführen zu können. Für Karl II. war das eine Existenzfrage, denn die Unklarheiten seiner Herrschafts-

1 Gottfried Seebaß, Apologia und Confessio, in: Martin Brecht/Reinhard Schwarz (Hg.), Bekenntnis und Einheit der Kirche, Stuttgart 1980, 9-21.

2 Heute: Karlsruhe-Mühlburg. 
rechte in Südbaden waren von den katholischen Habsburgern, also dem Kaiserhaus, stets in Erinnerung gebracht worden. Der Markgraf durfte dem Kaiser auch nicht den geringsten Anlass geben, seine Ansprüche in den Herrschaften Rötteln, Hochberg und Sausenberg in Frage gestellt zu sehen.

Die Grundlage der badischen Reformation waren also die CA und Melanchthons Apologie (also: Verteidigung der CA) - dazu traten die Schmalkaldischen Artikel Luthers, ein Zugeständnis an die sächsischen Theologen. Ansonsten setzten sich im badischen Reformationswerk die Württemberger Theologen durch.

Karl folgte weiterhin dieser südwestdeutschen Allianz, auch als Württemberg und Kurpfalz mit Kursachsen und anderen 1558 einen Versuch wagten, die Einheit des Protestantismus mittels eines durch und durch melanchthonisch geprägten Dokuments herzustellen. ${ }^{3}$ Dieses Dokument war der sog. Frankfurter Rezess, den auch Karl II. unterschrieben hat. Seit dem Übergang der Kurpfalz zum Reformiertentum nach 1559 zerbrach diese Allianz und wich bitterster Polemik zwischen Tübingen und Heidelberg. ${ }^{4}$

Nun wird es komplizierter: Im (heute) mittelbadischen Zentralterritorium wirkten überwiegend aus Württemberg stammende Theologen aus der Schülerschaft der Reformatoren Johannes Brenz (1499-1579) und Jakob Andreae (15281590). Baden selbst hatte ja kaum evangelische Pfarrer; Württemberg stellte diese Pfarrer für den westlichen Nachbarn. Und auch der Generalsuperintendent in Pforzheim, Ruprecht Dürr (ca. 1525-1580), stammte aus Württemberg.

Die kleine badische Markgrafschaft war viel zu unbedeutend, dass man die Koalition mit Württemberg hätte aufkündigen können. Dennoch gibt es Anzeichen, dass Baden dem württembergischen Weg hin zur Konkordienformel, der orthodoxen Festigung des Luthertums nicht uneingeschränkt folgte. Wieder sind es zwei Sachverhalte, die hier zu nennen sind: Zum einen war es der eben genannte Ruprecht Dürr, der in einem Gespräch in Maulbronn 1576, bei dem die Vorlagen zur Konkordienformel (FC) gesichtet wurden, Formulierungen in der Abendmahlslehre stützte, die milde ausfielen und sich nur an der CA, der Apologie und der Wittenberger Konkordie von 1536 orientierten: die sog. Maulbronner Formel $^{5}$, die Ruprecht Dürr für Baden unterschrieb. Zum zweiten war man sich in Durlach darüber im Klaren, dass man vorsichtig verfahren musste, denn in den südbadischen Herrschaften gab es starken reformierten Einfluss durch die nahe

3 Irene Dingel, Melanchthons Einigungsbemühungen zwischen den Fronten: der Frankfurter Rezeß, in: Jörg Haustein (Hg.), Philipp Melanchthon. Ein Wegbereiter für die Ökumene, Göttingen 1997 (Bensheimer Hefte 82), 119-141.

4 Vgl. dazu Julia D. Weiß, Admonitio Christiana, Stuttgart 2018, passim.

5 Titel, Kommentar und Text in BSELK QuM 2, 277-340. 
Schweiz. Generalsuperintendent in diesen Gebieten war in Personalunion der Basler Antistes Simon Sulzer (1508-1585), ein Schüler Martin Bucers (1491-1551).

Sulzer hat zwar die Unterschrift der badischen Pfarrer unter die FC gutgeheißen. Aber sein Abendmahlsbekenntnis von $1578^{6}$ bietet eine oberdeutsche Theologie im Lichte Bucers und auch Nähe zu Melanchthon - jedenfalls nicht die Lehre des Konkordienluthertums.

Hier ist nun ein Rückblick nötig: Nicht zuletzt aus politischen Gründen und zur Stärkung protestantischer Einheit waren 1536 Wittenberger Theologen und Theologen aus dem Oberdeutschen Raum (Straßburg, Esslingen, Reutlingen, Augsburg, aber einer auch aus dem Kraichgau) in Wittenberg zusammengetroffen und hatten ein Communiqué zur Abendmahlslehre unterzeichnet - die bereits genannte Wittenberger Konkordie. ${ }^{7}$

Und wieder sind zwei Linien zu verfolgen. Einmal: Die Bedeutung der CA für die Protestanten im Reich wuchs, indem bei Friedensverhandlungen 1532 und 1539 die CA als Positionspapier der Evangelischen zugrunde gelegt wurde.

Die zweite wichtige Linie ist diese: Im Jahre 1540 nahm Philipp Melanchthon im Vorfeld des Regensburger Religionsgesprächs 1541 eine Redaktion der CA vor, wobei er den Abendmahlsartikel 10 kürzte und mit dem Wörtchen CUM (MIT) das Abendmahlsgeschehen als ein Zusammenspiel göttlichen Wirkens und menschlichen Handelns entfaltete. Melanchthon arbeitete das Ergebnis der Wittenberger Konkordie in die aktualisierte Ausgabe der CA ein - und er veränderte damit faktisch die CA. Damals fand das keinen Anstoß; später schon, wie wir noch sehen werden.

Erfolgte 1540 also eine Redaktion der CA, so entwarf 1551 Melanchthon für das Konzil von Trient ein kursächsisches Bekenntnis, die confessio saxonica. ${ }^{8}$ Auch hier arbeitete er seine Abendmahlslehre ein. Wichtiger aber noch als dieser Umstand ist die Tatsache, dass diese neuerliche kursächsische Konfession mit dem Anspruch auftrat, eine repetitio, eine Wiederholung der CA zu sein. ${ }^{9}$ Die CA war jetzt also normierende und legitimierende Tradition.

Und ebenso war es der vorhin schon genannte Frankfurter Rezess, der nicht nur diese melanchthonische Abendmahlslehre enthielt, sondern wiederum sich als repetitio der CA verstand. An der CA kam man nicht vorbei und wollte das auch

\footnotetext{
6 Jetzt abgedruckt und erschlossen bei Daniel Abendschein, Simon Sulzer, Stuttgart 2019, 509543.

7 Abdruck in BDS 6, 1, 121-131 und RBS I, 2, $86 \mathrm{f}$.

8 MSA 6, 80-167.

9 Vgl. dazu Günther Wartenberg, Die Confessio Saxonica von 1551 und ihre Außenwirkung, in: Günter Frank/Stephan Meier-Oeser (Hg.), Konfrontation und Dialog. Philipp Melanchthons Beitrag zu einer ökumenischen Hermeneutik, Leipzig 2006, 219-234.
} 
nicht. Dennoch: In wesentlichen Punkten wurde nun das als CA ,wiederholt“, was in den Augen des konsequenten Luthertums nicht als Lehre der CA gelten konnte. Damit wurde die CA in ihrer Gültigkeit weiter bestätigt. Zugleich kam es aber zum Streit der Interpretationen vor allem in der Abendmahls- und Rechtfertigungslehre. Jeder berief sich auf die CA, sowohl die Anhänger Melanchthons wie auch das Luthertum Weimarer oder norddeutscher Prägung. ${ }^{10}$

Jetzt aber - wir wählen das Jahr 1559 - spaltet sich die Wirkungsgeschichte der CA in eine dreifache auf: eine melanchthonische, eine reformierte und eine konsequent lutherische, eine teilweise bis heute den Protestantismus belastende Entwicklung.

(1) Zunächst die melanchthonische bzw. philippistische Perspektive: Kurz vor Melanchthons Tod (1560) erschien noch eine Sammlung von Lehrschriften (Corpus doctrinae ${ }^{11}$ ), in der gleich in mehreren Schriften Melanchthon seine Abendmahlslehre vortrug, die er als Fortentwicklung, keineswegs als Zurücknahme der CA ansah. So konnte er - polemisch betrachtet eine Irreführung - im Inhaltsverzeichnis und Vorwort (der lateinischen Ausgabe) von der CA sprechen, die 1530 dem Kaiser Karl übergeben worden war. Der Text war freilich der von 1540 mit den entsprechenden Änderungen in der Abendmahlslehre. Entsprechend ergab sich eine Traditionslinie: CA 1530, Wittenberger Konkordie 1536, CAvar 1540, Corpus doctrinae 1560.

(2) Dann die reformierte Entwicklung: $\mathrm{Zu}$ den Abendmahlsstreitigkeiten zwischen lutherischen und reformierten Theologen in Heidelberg hatte Melanchthon im Herbst 1559 ein Gutachten erstellt, in dem er erneut seine Abendmahlslehre vortrug. ${ }^{12}$ Für die jetzt vom Luthertum zum Reformiertentum drängende Kurpfalz bedeutete dieses Gutachten zweifellos einen maßgeblichen Zwischenschritt. Bereits die lutherische Kirchenordnung des Kurfürsten Ottheinrich von 1556 war durch Melanchthons Examen Ordinandorum (1553) $)^{13}$ ergänzt worden, eine Schrift, die ebenfalls die typisch melanchthonische Abendmahlstheologie enthielt und die auch ins Corpus doctrinae aufgenommen worden war. Der 1563 erscheinende Heidelberger Katechismus war nun zweifelsfrei nicht mehr lutherisch, vielleicht eben noch melanchthonisch in seiner Abendmahlslehre, zugleich aber auch schon kalvinisch. Nichtsdestotrotz waren in einer Verteidigungsschrift von 1564 die Heidelberger reformierten Theologen fest davon überzeugt, dass in ihrer Abendmahlslehre Bibel, altkirchliche Rechtgläubigkeit

10 Zunächst fassbar im Magdeburger Bekenntnis von 1550: Bekentnis Vnterricht vnd vermanung der Christlichen Kirchen zu Magdeburgk. Anno 1550.

11 Vgl. MSA 6, Ziff. I. - VI.

12 MSA 6, Ziff. XI.: Iudicium de controversia de coena Domini [1559] 1560, 482-486.

13 MSA 6, Ziff. IV.: Examen ordinandorum [...] 1553, 168-259. 
und die CA zur Geltung kämen. ${ }^{14}$ Aus Sicht der Heidelberger reformierten Theologen ergab sich also eine Wirkungsgeschichte der CA, die man mit den Stationen CA 1530, CAvar 1540, Frankfurter Rezess 1558 ${ }^{15}$, Heidelberger Katechismus 1563 beschreiben konnte. Für die Reformierten war dies von hoher Bedeutung, da an der Rezeption der CA die reichsrechtliche Legitimität des Reformiertentums hing. Es mag bei einem Ausgleichsversuch der protestantischen Stände auf dem Naumburger Fürstentag 1561 durchaus Erschrecken ausgelöst haben, als man feststellte, dass es tatsächlich verschiedene Fassungen der CA gab. Damals hat man die CA von 1540 (variata) freilich notgedrungen akzeptiert.

(3) Der lutherische Weg war ein anderer. Der Schock des Kurpfälzer Weges saß vor allem in Württemberg tief und führte zum Einigungsweg des Konkordienluthertums, an dem Jakob Andreae maßgeblich beteiligt war. Das ist hier nicht zu schildern. Festzuhalten ist, dass auch die Konkordienformel sich selbst wie selbstverständlich als legitime Wiederholung der CA verstand, nun aber definitiv der ungeänderten CA von 1530 unter Ausschluss abweichender Interpretationen. Zwar wurde auch die Wittenberger Konkordie aufgenommen, aber im Text quasi „absorptiv“ umrahmt.

Damit ist wieder der Horizont der badischen Geschichte erreicht.

\section{Der Streit der Brüder und dessen Vorgeschichte}

Wie bereits geschildert hatte der „mittelbadische“ Generalsuperintendent sich an den Vorarbeiten zur Konkordienformel beteiligt. Sein Bezugspunkt waren allein die CA und deren Apologie (nebst Wittenberger Konkordie). Das ist wichtig. Zum gewaltigen Problem wurde nun aber der frühe Tod des Markgrafen Karl 1577 mit sich daraus ergebenden drei Irritationen.

Die erste Irritation bestand darin, dass wegen der Unmündigkeit der Söhne Karls nun eine Vormundschaftsregierung eingesetzt wurde; die beiden wichtigsten Vertreter waren die kurzzeitig wieder lutherische Kurpfalz und der Vorreiter des Konkordienluthertums Württemberg. Vor allem der Regierung in Stuttgart war zu verdanken, dass die nun fällige Unterschrift der Pfarrer unter die FC im Mittelterritorium auf geringen, in Südbaden aber auf erheblichen Widerstand stieß. Die südbadische Pfarrerschaft bestand zu großem Teil aus Baslern, die Simon

14 Gründtlicher Bericht vom heiligen Abendmahl unseres Herren Jesu Christi aus einhelliger Lere der heiligen Schrifft der alten rechtgläubigen Kirchen und auch der Augspurgischen Confession. Gestellt durch der Universität Heydelberg Theologen, Heidelberg 1564.

15 Diesen hatte der Heidelberger Kurfürst Ottheinrich (reg. 1556-1559) mit initiiert und unterzeichnet. 
Sulzer vermittelt hatte. Er selbst unterstützte die Unterschrift seiner badischen Pfarrer, auch wenn er die Abendmahlstheologie der FC nicht geteilt haben kann. Aber er erkannte die reichsrechtliche Bedeutung. Basel selbst war damals zerstritten und bewegte sich zwischen dem Bucertum Sulzers und dem wachsenden Zürcher Einfluss, der vor allem durch die schwäbisch-basler Familie Grynäus ausgeübt wurde. Thomas Grynäus (1512-1564) war nun einer der ersten badischen Pfarrer und Superintendenten Basler Herkunft, ebenso sein Sohn Jakob (1540 1617), später Sulzers Nachfolger in Basel, und dessen Bruder Theophil (15341583), ein Melanchthonschüler.

Mit - die Zahlen schwanken - vielleicht sieben Kollegen wurde nun das Grynäische Pfarrhaus in Rötteln zur Zelle des Widerstandes gegen die FC. ${ }^{16}$ Die „Halstarrigen“ mussten schließlich gehen. Für uns ist wichtig zu sehen, dass die Widerständigen sich nicht etwa auf reformierte Bekenntnistraditionen beriefen, sondern auf die lutherisch-badische Kirchenordnung von 1556: d.h. (allein) die CA und deren Apologie. Dem seien sie verpflichtet, was hier stehe, sei ausreichend. Dahinter stand freilich die Überzeugung, dass die lutherische Fortbildung der Abendmahlslehre und der damit verbundenen christologischen Auffassungen als illegitim anzusehen seien. In der Aufnötigung der FC erblickten diese Pfarrer eine Veränderung des badischen Bekenntnisstandes!

Wie dem auch sei, seit der Unterschrift der Vormünder für die badisch-durlachischen Prinzen im November 1579 war das Territorium nun offiziell dem Konkordienluthertum zugehörig.

Die folgenden zwei Irritationen erwuchsen aus der Mündigkeit der beiden älteren Prinzen Ernst Friedrich und Jakob (1584).

(1) Nach zwei Religionsgesprächen trat Jakob III. (1562-1590) ${ }^{17} 1590$ zur römisch-katholischen Kirche über. Ob er dies unter Beratung des zunächst lutherischen, dann reformierten, schließlich katholischen Arztes und Rates Johann Pistorius (1546-1608), Sohn des gleichnamigen hessischen Reformators, ${ }^{18}$ getan hat, oder politische Rücksichten maßgeblich waren, ist bisher leider nur Gegenstand polemischer Forschung gewesen. Ein wichtiger Hinweis könnte aber darin liegen, dass der fortwährende Streit im Protestantismus, der ja zwischen 1577 und 1617 nicht endete, mit seiner Vorstellung einer kirchlichen Einheit nicht vereinbar

16 Vgl. dazu einstweilen Friedemann Merkel, Geschichte des evangelischen Bekenntnisses in Baden von der Reformation bis zur Union, Karlsruhe 1960, 43-49.

17 Helmut Steigelmann, Die Religionsgespräche zu Baden-Baden und Emmendingen 1589 und 1590, Karlsruhe 1970.

18 Hans-Jürgen Günther, Die Reformation und ihre Kinder - Vater und Sohn Johannes Pistorius Niddanus - eine Doppelbiographie, in: Niddaer Geschichtsblätter 2 (Selbstverlag Nidda 1994). 
war. Kurz nach seiner Konversion starb Jakob III. an einer Arsen-Vergiftung; das bietet bis heute Anlass zu nicht immer ökumenischen Spekulationen.

(2) Das Erbe seiner südbadischen Herrschaften trat sein älterer Bruder Ernst Friedrich (1560 - 1604) an. Dessen zeitweiliger Hauslehrer und Prinzenerzieher war Georg Hanfeld (ca. 1545 - nach 1595) gewesen, der 1579 am reformierten Casimirianum in Neustadt wirkte und sein pastorales und schriftstellerisches Wirken nach seiner Pfarrtätigkeit an der Heidelberger Peterskirche (1585-1590) als Superintendent in Bretten beschloss. Er und vielleicht Pistorius in seiner reformierten Phase weckten in Ernst Friedrich erhebliche Zweifel an der Legitimität des Luthertums in seiner jüngsten Entwicklung. Von der Unterschrift seiner Vormünder distanzierte sich der nunmehrige Regent. Mehr noch: er suchte durch voluminöse Schriften die Legitimität der Konkordienformel zu bestreiten. Mit dem Exemplar der CA seiner Kanzlei und sogar Luthers Großem Katechismus erkannte er darauf, dass in der FC zu Abendmahl und Christologie nur ungereimte Dinge stünden. Erster Referenzpunkt war also seine CA aus der Durlacher Kanzlei, die er irrend für einen Originaldruck hielt. Die Kritik an der FC sollte den Übergang zum Reformiertentum legitimieren. Es gibt leider nur wenige Hinweise auf kurpfälzisch-badische Kooperation. Es fällt aber auf, dass Ernst Friedrichs reformiertes Bekenntnis - das sog. Stafforter Buch von $1599^{19}$ - nach dem Ort benannt ist, in dem es gedruckt wurde. Und Staffort gehört zu den altbadischen Orten, die Heidelberg oder auch dem kurpfälzischen Weingarten räumlich am nächsten lagen. War der Druckort also der Ermöglichung einer unauffälligen „kurpfälzischen Redaktion“ geschuldet? Wenige Jahre später erfolgte ein Nachdruck des Buches in Heidelberg und in Neustadt - jeweils vermehrt durch eine Verteidigungsschrift der kurpfälzischen Theologen gegen ${ }^{20}$ die umfassende Bekämpfung des Stafforter Buches durch Kursachsen und Württemberg. ${ }^{21}$

19 „Christlichs Bedencken“ und „Kurtze vnd Einfeltige ausser Gottes Wort vnd der Alten Rechtglaubigen Kirchen gestelte Bekandnuß, Staffort 1599“.

20 Deß Durchleuchtigen Hochgebornen Fürsten vnd Herrn / Herrn Ernst Friderichen [...] Wolgegründte vnd satte Ableinung / der / durch die Theologen deß Württembergischen Consistorij zu Stuttgarten / wider Jhrer F. Gn. Christliches Bedencken vnd erhebliche wolfundierte Motiven [...] welche in Jhrer F.G. Landt heimlicher weis eyngescheucht worden [...] Gedruckt zur Newstadt an der Hardt / Anno M. D CI. [= Neuauflage des Staffortschen Buches mit Apologie (Zusatz)]; (Anonymus, Heidelberger Theologen) Kurtzer Beweiß/ Das des Durchleuchtigen Hochgebornen Fürsten vnd Herren/ Herren Ernst Fridrich Marggrafen zu Baden vñ Hochberg/ Landgrafen zu Sausemberg Herrn zu Röteln vnd Badenweiler ec. Christliche Confession in dem grossen Tübingischen Buch nicht widerleget. Heydelberg Im Jahr 1601.

21 [Kursachsen:] Gründtliche Widerlegung Des zu Staffort / vnter dem namen / des Durchleuchtigen Hochgebornen Fürsten vnd Herrn / Herrn Ernst Friedrichen / Marggraffen zu Baden etc. Anno 99. außhesprengten Caluinischen Buchs [...] Durch die Churf. Sächsische hiezu ver- 
Auch dieser kirchenpolitisch riskante Versuch scheiterte, zunächst am Widerstand der Pforzheimer Bürgerschaft ${ }^{22}$ und dann durch den frühen Tod des Markgrafen schon 1604.

Das Gesamterbe trat nun Georg Friedrich (1573-1638; resign. 1622) an, bekannt als der Verlierer der Entscheidungsschlacht im Südwesten bei Wimpfen im Dreißigjährigen Krieg (1622). Georg Friedrich konsolidierte erneut die badische Kirche als Kirche konkordienlutherischer Prägung. Erst mit ihm ist eigentlich die lutherische Reformationsgeschichte Altbadens abgeschlossen.

Freilich sind auch hier zwei Sachverhalte auffällig: (1) Als Kriegsherr stellte er die Bekenntnisfrage im Gegensatz zu Württemberg klar zurück, war und blieb (auch nach Übergabe der Herrschaft an seinen Sohn 1622) engster Verbündeter Kurfürst Friedrichs V. von der Pfalz. Georg Friedrich selbst starb in Straßburg. (2) Die kirchliche Reorganisation der Markgrafschaft Baden-Durlach nach dem Westfälischen Frieden 1648 erfolgte lediglich mit einem Neudruck der alten Kirchenordnung Karls durch Markgraf Friedrich V. Erst seine Vorrede zur 1649 erneut und in Straßburg nachgedruckten Kirchenordnung kennt einen expliziten Bezug zur FC und zur ungeänderten CA. ${ }^{23}$ Einzug gehalten hat die Konkordienformel auch in die Ordinationsverpflichtung, wohl das ganze 17. und auch das 18. Jahrhundert, aber in eher blasser Gestalt, wenn es heißt, ,also sollen die Prediger sich [...] befleißigen, ihrem Amt ein Genügen zu leisten, und zu dem Ende das Wort Gottes

ordnete Theologen [...] Wittemberg / [...] Anno 1602; [Württemberg:] Kurtzer vnd warhaffter Bericht AVff zwo vnterschidliche/ vnter dem Namen des Durchlauchtigen Hochgebornen Fürsten [...] Ernst Friderichen/ Margrauen zu Baden [...] publicirte Schrifften: damit deroselben Verfasser dem bewußten Staffortischen Buch auf die Bein zuhelffen / vnd es wider der Würtembergischen Theologen Summarische Relation, vnd daruff geuolgte außführliche Widerlegung ermelten Staffortischen Buchs / zuuertheidigen / dardurch auch des Caluinismus sich zuentladen / vergeblich vnterstanden. Auff Gnädigen Beuelch / Des Durchleuchtigen Hochgebornen Fürsten vnd Herrn / Herrn Friderichen / Herzogen zu Würtemberg [...] ec. Gestellt Durch die hierzu verordnete Würtembergische Theologen. Tübingen Anno M. DCII.

22 Volker Leppin, Der Kampf des Markgrafen Ernst Friedrich von Baden um sein Bekenntnis und der Widerstand aus Pforzheim, in: Udo Wennemuth (Hg.), Reformierte Spuren in Baden, Karlsruhe 2001, 52-67.

23 Kirchen-Ordnung/ Sampt anderen Mandaten/ Wie die in der vndern Marggraffschafft Baden/ Marggraffschafft Hochberg/ Landgraffschafft Sausenberg/ Herrschafft Röteln vnd Badenweyler/ solle gehalten werden. Gedruckt zu Straßburg/ bey Johann Andreae Sel. Erben / Anno 1649, ii a: „Und aber wir in vnserer Regierung biß dahero/ nach den löblichen Fußstapfen vnserer Gottseeligen Vorfahren/ die wahre vnd allein seeligmachende Lehr des H. Evangelij/ nach anleitung der vngeänderten Augspurgischen Confession vnd formule Concordię bey vnsern getrewen Vnterthanen vnserer Fürstenthumb vnd Landen/ auff die liebe Nachkommen fortzupflantzen vnd vnverruckt zu erhalten $[. .$.$] “.$ 
und die aus demselben gezogene symbolische Bücher, so in der Formula Concordiae begriffen seynd, und andere geistreiche Schrifften fleißig zu lesen.“24

Wir können also innerhalb der badischen Kirchengeschichte von einer mehrfachen Bezugnahme auf die CA sprechen, zunächst die Grundlegung in der badischen Kirchenordnung von 1556, dann ihre Rezeption im Sinne des Konkordienlutherums nach 1577; ihre Verwerfung durch Jakob III., ihre reformierte Vereinnahmung unter Ernst Friedrich und schließlich ihre (erneute) Bekräftigung im Sinne des Konkordienluthertums durch Georg Friedrich.

\section{Die Confessio Augustana als inspiriertes Wort Gottes 1730}

1630 wurde das erste Augustana-Jubiläum in Baden begangen. Es kann kein Zweifel herrschen, dass in Baden nach dem großen Krieg eine lutherische Orthodoxie Einzug hielt. Allerdings wird diese in Kirchenordnungen nicht (mehr) sichtbar. Schon seit 1629 traten neben die Kirchenordnung und nach 1649 faktisch an deren Stelle sog. Synodalbefehle, die zwar immer von rechter Lehre sprachen, dies aber nicht entfalteten. Als Hauptliteratur galten nun Johann Arndts vier bzw. sechs Bücher vom wahren Christentum sowie dessen Paradiesgärtlein ${ }^{25}$. Wichtige aus Baden stammende Theologen wirkten im lutherisch-orthodoxen Sinne v. a. in Straßburg [z.B. Johann Georg Dannhauer (1603-1666), der Lehrer Speners], die folgende Generation wich aufgrund der Kriegsverwüstungen nach 1689 auf auswärtige Hochschulen aus [z.B. der hochorthodoxe Johannes Fecht (1636-1716) nach Rostock].

Allerdings - ein ganzer wesentlicher Aspekt - wird man auch auf die sog. Augustanafeiern zu blicken haben. Sie stehen nicht nur für die Bedeutung der CA für eine Kirche, sondern machten das Grundbekenntnis auch zum Gegenstand der öffentlichen Feier, also „volkstümlich“.

Als 1717 die Karlsruher Schlosskirche eingeweiht wurde, wurde dies verbunden mit der Feier des Reformationsjubiläums, das bereits 100 Jahre zuvor begangen worden war. Die Indienstnahme der Kapelle war verbunden mit dem

24 Kirchen-Agenda, Carlsruhe 1750, 271.

25 Vgl: Johann Arnd's des hocherleuchteten Lehrers, weil. General-Superintendenten des Fürstenthums Lüneburg Sechs Bücher vom wahren Christenthum, nebst dessen Paradies-Gärtlein. Mit der Lebensbeschreibung des seligen Mannes [...] Neue Stereotyp-Ausgabe. Fünfter Abdruck. Stuttgart [o.J.; Original $1605 \mathrm{f}$ und 1617]. 
feierlichen Eintragen der Bibel, der Sakramentsgeräte, der Agenden - und des Konkordienbuches (das aber, wenn ich recht sehe, in Baden nie gedruckt wurde)! Bei dieser Feier wurde nun ein Gebet vorgetragen, das - ein Initial der späteren Entwicklung - die Reformation ganz auf die Übergabe der CA an den Kaiser bezog. Hier heißt es u.a.:

$\mathrm{Du}$, Gott, hast uns abermal einen Tag erleben lassen, den du Herr gemacht hast, darinnen wir uns freuen und fröhlich sein und an demselben uns billich mit dankbarem Herzen erinnern sollen deren überaus großen und hohen Gutthaten, die du anjetzo vor 2 hundert Jahren deiner höchstbedrängten und durch die Päpstische Abgötterei und Greuel jämmerlich verführten Christenheit reichlich erzeiget: in dem du nicht allein das selige Licht deines heiligen Worts, welches eine lange Zeit verdunkelt geblieben, durch deinen getreuen Diener und auserwähltes Werkzeug D. Luthern wunderlich angezündet und erleuchtet, sondern eine kleine Zeit hernach unter dem grausamen Wüten und Toben des Satans und fast der ganzen Welt, etliche wenige christliche Obrigkeiten erwecket hast, die mit Gottseligem Eyfer und tapffrem unerschrockenen Heldenmuth die ganze reine und alleinseligmachende Lehre in eine kurze Schrift zusammengezogen, sich mit Mund, Hand und Herzen zu solcher bekandt, und dieselbige auf dem großen Reichstag in Augspurg übergeben, öffentlich abgelesen und hiermit deinen Nahmen und dein heiliges Wort vor Kayser, König, Fürsten und Herren usw. damahlen getragen haben. Du hast aber, o großer und starker Gott noch ferner diesen deinen wunderbarlichen Segen verliehen, daß von dieser Reichsversammlung und solch Christl. Evangel. Glaubensbekäntniß in unterschiedlichen Sprachen bei vielen anderen Ländern und Völkern in der Welt bekandt gemacht und bald darauf auch dieser Marggrafschaft der gute Geruch deines Erkäntniß und heiligen Evangelii durch deine Göttliche Kraft geoffenbaret worden, welchem gemäß in diesen Fürstenthumen und Landen auf diese Stund gelehret wird. $^{26}$

Es folgen noch ein paar Unfreundlichkeiten gegen Katholiken und Calvinisten. Klar: Luther musste genannt werden. Aber wesentlich war das Bekenntnis von 1530 durch die christlichen Obrigkeiten, sowie die Ausbreitung der CA.

Gesteigert wurde die Bedeutung der CA dann im Jubiläumsjahr 1730. Noch gemäßigt zeigten sich die dazu erörterten Thesen des Pforzheimer Pfarrkonvents, ${ }^{27}$ die sich so zusammenfassen lassen: Die CA ist aus Antrieb des Heiligen Geistes von den Protestanten aus den Heiligen Schriften besiegelt: zur Wiedergabe des Glaubens und zur Verteidigung gegen die Verleumdungen der Gegner. Sie darf das erste und vorzügliche Symbol der lutherischen Kirche genannt werden. Andere Symbole wie die Apologie oder die Schmalkaldischen Artikel sind ihr nachgeordnet. Sie ist der heiligen Schrift aber nicht gleich.

26 Zitat nach Emil Zittel, Das Reformationsjubiläum von 1817 und Die Union, Heidelberg 1897, 3. 27 Vgl. dazu und zu den folgenden Durlacher Thesen: Johannes Bauer, Zur Geschichte des Bekenntnisstandes der vereinigten ev.-prot. Kirche im Großherzogthum Baden, Heidelberg 1915, 5-7. 
Der Durlacher Konvent zeigte sich freilich maßlos, wenngleich der Religionseid auf die Bekenntnisse dort auch auf Widerstände stieß, wofür man „Pietisten“ verantwortlich machte. Dort hieß es: Die CA wird mit Recht Glaubensbekenntnis genannt. Ihr höchster Autor und hauptsächlicher Urheber ist der dreieinige Gott selbst. Sie ist Norm, u.z. unfehlbare. Sie muss absolut, nicht conditional unterschrieben werden. Sie bietet keinen Anknüpfungspunkt zu den Reformierten, allenfalls die Möglichkeit eines weltlichen Friedens mit ihnen. (Damit stellte man sich auf den Standpunkt der Tübinger Theologen von 1616!) ${ }^{28}$ Melanchthons CA von 1540 stellt einen substantiellen Texteingriff zugunsten des Zwinglianismus dar, ist also zu verwerfen. Und schließlich: Gottfried Arnolds (1666-1717) polemische Auffassung, ${ }^{29}$ die CA bilde ein (evangelisches) Papsttum, ist grundfalsch.

Schärfer, polemischer und kirchenpolitischer ist in Baden die CA kaum mehr betrachtet worden. Ein halbes Jahrhundert später schon kannte die badische Kirche auch keinen Religionseid mehr. Nachdem dieser 1786 letztmals erneuert wurde, wurde er bereits 1788 abgeschafft.

\section{Die Geltung der Confessio Augustana als Grundlegung der Unionskirche 1821}

Die Geschichte des Werdens der badischen Union ist hier nicht zu schildern. ${ }^{30}$ Erwähnenswert ist aber, dass sie ohne das Wirken des Juristen Friedrich Brauer $(1754-1813)^{31}$ nicht denkbar ist. Seine multiplen Integrationsmaßnahmen waren von der Auffassung geleitet, dass eine Unionskirche (die Union selbst hat er nicht mehr erlebt) aufgrund ihrer Neubildung nicht den Rechtstatus des alten Reiches verlieren dürfe, sich also auf die CA gründen müsse.

Scheinbar selbstverständlich wurde die CA zum Grundbekenntnis der badischen Union. Scheinbar deshalb, weil im Vorfeld der Unionsberatungen die CA durchaus als lutherisches Bekenntnis verstanden wurde, der ergänzend der Heidelberger Katechismus zur Seite gestellt werden sollte. Es hat sich dann aber eine Formulierung durchgesetzt, welche die CA als verbindendes Grunddokument erwähnte und speziellen konfessionellen Prägungen durch Aufnahme des Kl. Ka-

28 Vgl dazu wieder Julia D. Weiß, Admonitio christiana, 229-234.

29 Eine Anspielung auf dessen Unpartheyische Kirchen- und Ketzerhistorie (1699/1700).

30 Vgl. dazu: Johannes Ehmann, Union und Konstitution. Die Anfänge des kirchlichen Liberalismus in Baden im Zusammenhang der Unionsgeschichte (1797-1834), Karlsruhe 1994.

$31 \mathrm{Zu}$ Brauer allgemein vgl.: Christian Würtz, Johann Niklas Friedrich Brauer (1754-1813). Badischer Reformer in napoleonischer Zeit, Stuttgart 2005. 
techismus Luthers und des Heidelberger Katechismus Genüge tat. Damit begriff man nun die CA als mit dem Luthertum der alten Markgrafschaft und dem Reformiertentum der rechtsrheinischen Kurpfalz vereinbar - keineswegs eine Selbstverständlichkeit. ${ }^{32}$

Für unser Thema hochinteressant ist die Deutung der CA in der Vereinigungsurkunde (§ 2):

Diese vereinigte evangelisch-protestantische Kirche legt den Bekenntnisschriften, welche späterhin mit dem Namen symbolische Bücher bezeichnet wurden, und noch vor der wirklichen Trennung in der evangelischen Kirche erschienen sind, und unter diesen namentlich und ausdrücklich der Augsburgischen Konfession im allgemeinen, sowie den besonderen Bekenntnisschriften der beiden bisherigen Evangelischen Kirchen im Großherzogtum Baden, dem Katechismus Luthers und dem Heidelberger Katechismus das ihnen bisher zuerkannte normative Ansehen auch ferner [bei] [...].

Soweit können wir gut folgen. Der Text fährt nun signifikant für den neuzeitlichen Geist fort:

insofern und insoweit [...], als durch jenes erstere mutige Bekenntnis vor Kaiser und Reich das zu Verlust vergangene Prinzip und Recht der freien Forschung in der heiligen Schrift als der einzig sicheren Quelle des christlichen Glaubens und Wissens wieder laut gefordert und behauptet, in diesen beiden Bekenntnisschriften [Kleiner Katechismus Luthers, Heidelberger Katechismus] aber faktisch angewendet worden, demnach in demselben die reine Grundlage des evangelischen Protestantismus zu suchen und zu finden ist. ${ }^{33}$

Dieses ,insofern und insoweit“ ist in der Jahrhundertmitte erwartungsgemäß zum Streitpunkt geworden. Der Deutung der CA 1821 liegt nun aber folgendes Verständnis zugrunde: Die CA ist das Grundbekenntnis des Protestantismus, nämlich das Bekenntnis zur freien Schriftforschung, und bildet somit (!) das übergeordnete Prinzip, von dem aus die beiden Konfessionskatechismen praktisch abgeleitet werden.

Das ist historisch ein wenig schief, da Luthers Katechismen ja schon vor der CA entstanden sind. Auch würde man das Schriftprinzip eher mit der Leipziger Disputation schon von 1519 verbinden. Wichtig war aber das Bekenntnis vor Kaiser und Reich als protestantisches Prinzip. Die spätere (1855) Bekräftigung des $\S 2$ im Sinne einer positiven Union unterstrich dem gegenüber die CA als

32 Vgl. dazu: Johannes Ehmann, Zwischen Konfession und Union. Zur Diskussion über die Vereinbarkeit von Heidelberger Katechismus und Augsburger Bekenntnis, in: EvTh 72,6 (2012), 457-465.

33 Zitat nach: Bekenntnisschriften der Evangelischen Landeskirche in Baden I, Karlsruhe 2014, $134 \mathrm{f}$. 
Grundlage der evangelischen Kirche Deutschlands und die Übereinstimmung mit den Grundlehren der Heiligen Schrift „und des in den allgemeinen Bekenntnissen der ganzen Christenheit ausgesprochenen Glaubens“34 - eine nationale und zugleich ökumenische Öffnung.

\section{Die Confessio Augustana als Konstitution des Erwecktentums 1830 und Inbegriff der Reformation}

Die erste landesweite Reformationsfeier der Unionskirche fand am 27. Juni 1830 statt - also nicht am 31. Oktober, sondern als Augustanajubiläum. Zwei Impulse sind kurz zu nennen.

(1) Mit dem Jubiläum setzte die badische Erweckung Aloys Henhöfers (17891862) und seiner Schüler einen ersten Markstein als künftiger „Player“ der badischen Kirchen- und Frömmigkeitsgeschichte. Ihnen war die Deutung der CA in der Unionsurkunde zu schwach.

Am 27. Juni 1830 schloss der erweckte Georg Adam Dietz in Friedrichsthal seine Predigt mit den Worten:

[...] [Es] ist [...] der heutige Tag das zweite [nach 1817] Signal zum Sammeln unter der Kreuzesfahne, die Reihen zu schließen. - Wir heißen Protestanten, aber nicht weil wir gegen, sondern für das Evangelium protestieren. Nicht weil gegen Zeitirrtümer protestiert wurde ist der Tag zu Augsburg ein hochwichtiger Tag, sondern weil die ewige Wahrheit, das Evangelium von Christo öffentlich bekannt wurde. Schämen müssen sich alle in ihr Herz hinein, denen das Evangelium Aergerniß, Thorheit oder ein Märlein ist; sie sollten sich heute in den Boden hinein verkriechen. Denn der heutige Ehrentag der treuen Bekenner ist der Tag ihrer Schmach und Schande, ihres Gerichts, und ein Vorbote dessen, was ihrer wartet am großen Tage. [...] Das sei unsre Losung: ,Hie Schwert des Herrn und Gideon!' der rechte Gideon Jesus Christ, der Herr Zebaoth und ist kein andrer Gott, das Feld muß er behalten! Amen. Amen Amen! $!^{35}$

Die CA war nun Bekenntnis des Evangeliums, an dem im Jüngsten Gericht Schafe und Böcke geschieden wurden.

(2) In gewisser Anlehnung an den Konstitutionsgedanken des Vormärz wurde darüber hinaus im ebenfalls 1830 einsetzenden badischen Katechismusstreit die

34 Bekenntnisschriften der Evangelischen Landeskirche in Baden I, 141.

35 Zitat nach: Geschichte der badischen evangelischen Kirche seit der Union in Quellen, Karlsruhe 1996, Nr. 38. 
CA von Henhöfer als „Geschäftsvertrag, Vertragsurkunde, Konstitution“ gegen Spätaufklärung und Frühliberalismus entwickelt. Die Confessio Augustana war zur kirchenpolitisch wirksamen Constitutio Augustana geworden. ${ }^{36}$

Eine von allen Gruppierungen gern getragene Entscheidung war die der Generalsynode von 1834, das jährliche Reformationsfest am letzten Junisonntag zu begehen. Wieder und weiterhin wurde also die Reformation mit der CA verbunden. Und - in ökumenisch durchaus erhitzter Zeit - hört man staunend die Bestimmung der Kirchensektion, es solle bei den Feiern „niemals die brüderliche Liebe gegen unsere Schwesterkirche vergessen werden, so daß alles mit christlicher Liebe geschehe. ${ }^{\text {“37 }}$

\section{Johannes Bauers Reklamierung der Confessio Augustana für die Unionskirche}

Es zwingt uns die Zeit zu einem großen Sprung, der manches Erwähnenswerte im Streit um Bekenntnisfreiheit und Bekenntnisbindung übergehen muss. Wir springen aus dem 19. Jahrhundert ins Jahr 1930. Damals hat Heidelberger Universitätsprofessor Johannes Bauer (1860 - 1933) einen kurzen Artikel in der liberalen Kirchenzeitung geschrieben, der in seinem historischen Rückblick wie auch für seinen Standpunkt für sich selber spricht. Bauer schrieb 1930:

Vielleicht ist es neulich dem einen oder anderen aufgefallen, daß in der Ankündigung der Augustanafeier, die auch an unseren Kirchentüren angeheftet war, zu einer Feier der lutherischen Kirche und des Luthertums aufgefordert wurde. Darnach schien es, als ob die reformierten und unierten Kirchen von der Feier ausgeschlossen seien, als ob sie diese Feier nichts angehe. [...] Wir in Baden haben von dem Jahre 1834 an bis zum Jahre 1882 das Reformationsfest jeweils am letzten Sonntag im Juni, eben zur Erinnerung an die Uebergabe des Augsburger Bekenntnisses am 25. Juni 1530, gefeiert. [...] Ohne die Frage hier näher zu erörtern, inwieweit die Augustana im übrigen den Ansichten Luthers entsprach, wird man doch sagen müssen, daß damals, im Juni 1530, Ansichten, die später ,reformiert' genannt wurden, ausgeschlossen waren, daß also das Bekenntnis von Augsburg insofern mit Recht als ein ausschließlich ,lutherisches‘ zu bezeichnen ist. Die Oberdeutschen - nicht die Schweizer - haben sich bald nach 1530 aus politischen Gründen und unter dem Einfluß des Straßburger Reformators Martin Butzer den Ständen, die die Augustana unterschrieben

36 Vgl. dazu wieder Ehmann, Union und Konstitution, 295f. Zum Katechismusstreit: ders., Die badischen Unionskatechismen. Vorgeschichte und Geschichte vom 16. bis zum 20. Jahrhundert, Stuttgart 2014, 269-298.

37 Karl-Friedrich Vierordt, Geschichte der evangelischen Kirche in den Großherzogthum Baden 2, Karlsruhe 1858, 438. 
hatten, genähert und wurden daher 1536 [scil. durch die Wittenberger Konkordie] in deren Gemeinschaft aufgenommen. [...] Das ist nach einer Seite hin die Vorgeschichte des § 2 unserer badischen Unionsurkunde. Von den ersten Verhandlungen an haben besonders die Vertreter der reformierten Pfalz auf die öffentlich-rechtliche Bedeutung der Augustana hingewiesen. ${ }^{38}[. .$.$] Daß diese Anlehnung an die Augustana in jenen Jahren um der Rechts-$ sicherheit der unierten Kirche willen [...] notwendig war, braucht hier nicht näher begründet zu werden. Heute [1930] aber liegen gänzlich andere öffentlich-rechtliche Verhältnisse vor. Die Stellung der evangelischen Kirche im Staat hängt nicht mehr unmittelbar von ihrer Zugehörigkeit zu den Augsburger Glaubensverwandten ab. Trotzdem werden auch die Reformierten dankbar der A.C. gedenken, nicht wegen ihres Inhalts und nicht wegen ihrer Bedeutung im alten Deutschen Reich, sondern weil jenes Bekennen der Fürsten und Stände, der Laien und Theologen ein erstes freudiges Zeugnis für das Evangelium war: in Worms war Luther allein der Bekenner, in Augsburg war es eine Gemeinschaft von solchen, die ,mutig vor Kaiser und Reich` (§ 2 der Unionsurkunde) ihre Glaubensüberzeugung bekannten. ${ }^{39}$

Historisch korrekt ordnete Bauer also die CA in die ältere und neuere Kirchengeschichte ein. „Liberal“ war freilich seine Interpretation. Der Bekenntnischarakter war geschmälert, die Rechtskraft der CA als Rechtslast abgetan. Was blieb, war das zuversichtliche Bekennen, das 1717 schon den christlichen Obrigkeiten zugesprochen worden war - jetzt generalisiert, ja demokratisiert hinsichtlich einer Gemeinschaft von Theologen und Laien, die (gut protestantisch) kein Glaubensbekenntnis, sondern ihre Glaubensüberzeugung kund taten. Die CA war weniger Zeugnis als ihre Übergabe lebendige Zeugenschaft.

\section{Ergebnis}

Wir haben die Geschichte der CA in unserer Kirche verfolgt und ihre unterschiedliche Funktion kennen gelernt. Sie diente der Grundlegung lutherischen Kirchentums, sie war so etabliert wie in ihrer Deutung umstritten, war dem Wort Gottes nahezu gleichrangig und (nur noch) Bewährung des Schriftprinzips. Sie wurde Rechtsdokument und als solches dann auch obsolet. Sie war notwendiges Bekenntnis und das Gewissen mancher auch beschwerendes Dogma. Sie war also im Grunde alles und ist es bis heute geblieben - einerseits verbindlich, andererseits unbekannt.

Ist das eine Schwäche oder eine Stärke? Selbst Inbegriff des volkskirchlichen Pluralismus? Oder in ökumenischer Perspektive ein Friedensinstrument? Diese

38 Vgl. Johannes Bauer, Zur Geschichte des Bekenntnisstandes der vereinigten ev.-prot. Kirche im Großherzogtum Baden, Heidelberg 1915, Nr. 45-47!

39 Johannes Bauer, Ist das Augsburgische Bekenntnis nur ein Bekenntnis der lutherischen Kirche?, in: Süddeutsche Blätter für Kirche und freies Christentum 1930, Nr. 8, 77-79, 78 f. 
Fragen kann ich hier und heute nur stellen und keine Antworten wagen; bis auf eine:

Betrachte ich historisch die neuralgischen Eckpunkte der Geschichte meiner Kirche, so taucht die CA immer auf; betrachte ich diese Eckpunkte theologisch, so will ich doch sagen, dass die CA - mit und bei allem kritischen Potential, das ihr innewohnt - eine gute Begleiterin der badischen Kirche gewesen ist.

Am Anfang stehen Reformwille, theologische Durchdringung und Bekenntnis. Ohne diese drei wird kaum eine Kirche auskommen. Die CA bietet dafür ein anschauliches Beispiel. 
